\title{
BMC Gastroenterology reviewer acknowledgment 2015
}

Elaine Zhang

\section{Contributing reviewers}

The editors of BMC Gastroenterology would like to thank all our reviewers who have contributed to the journal in Volume 15 (2015).

Noorizan Abdul Majid
Malaysia

Thomas Abrams

USA

Florence Abravanel

France

Syeda Afroze

USA

Daniel Agardh

Sweden

Rakesh Aggarwal

India

Ferdinando Agresta

Italy

Sinead Aherne

Ireland

Jawad Ahmad

USA

Y Akutsu

Japan

Badr Al Bawardy

USA

J. Wesley Alexander USA

\author{
Alicia Algaba \\ Spain \\ Turki Alharbi \\ Saudi Arabia \\ Marco Allaix \\ Italy \\ Lee-Ann H. Allen \\ USA
}

\section{Gianfranco Alpini}

USA

Mary Anne Amalaradjou

USA

Kamal Amin

Saudi Arabia

Leif Percival Andersen

Denmark

Tiing Leong Ang

Singapore

Harry Antoniades

UK

Toru Aoyama

Japan

Christina Arnold

USA

\section{Zubin Arora \\ USA}

Serhat Avcu

Turkey

Matias Avila

Spain

Hasan Aydin

Turkey

A B

Germany

Hideo Baba

Japan

Oliver Bachmann

Germany

Haibo Bai

USA

Michael Bailey

USA

Gian Luca Baiocchi

Italy

Snigdha Banerjee

USA

Claudia Banzato

Italy

Correspondence: elaine.zhang@biomedcentral.com

BMC Gastroenterology, BioMed Central, 233 Spring Street, New York, NY

10013, USA 
Edward Barnes

USA Minor Outlying Islands

Anushka Baruah

USA

Mohammad Bashashati

Canada

Guido Basilisco

Jamaica

Fateh Bazerbachi

USA

Paul Beavis

Australia

Anu Behari

India

Ajay Belgaumkar

UK

Alberto Bellan

Italy

Alexander Berezin

Ukraine

Marie-Luise Berres

Germany

Govind Bhagat

USA

Kalyan Bhamidimarri

USA

Bhushan Bhole

India

Federico Biagi

Italy

Itxarone Bilbao

Spain

Louise Bill

Denmark

Camilla Böckelman

Finland

Bruno Bonaz

France

Guillaume Bouguen

France

Alex Boussioutas

Australia

Christopher Bowlus

USA
Charlène Brochard

France

Christine Budke

USA

Elisabetta Bugianesi

Italy

Jason Burkhead

USA

Nick Burr

UK

Carlo Caffarelli

Italy

Diego F Calvisi

Germany

Fabio Campanile

Italy

Jordi Camps

Spain

Yin Cao

USA

Rocco Cappellesso

Italy

Marco Carbone

Italy

Andrea Cariati

Italy

Bertrand Cariou

France

Siobhán Cashman

UK

Patricia Castelucci

Brazil

Phillipo Chalya

Tanzania, United Republic Of

Heyson Chan

Hong Kong

Michael Huw Chapman

UK

Hrushikesh Chaudhari

India

Chieh-Chang Chen

Taiwan

Jun Chen

China
Ming-Jen Chen

Taiwan

Wen-Chi Chen

Taiwan

Hsiu-Chi Cheng

Taiwan

Giuseppe Chiarioni

Italy

Thirunavukkarasu Chinnasamy India

Sakkarin Chirapongsathorn

Thailand

King-Wah Chiu

Taiwan

A Cho

Japan

Evangelos Cholongitas

Greece

Bruno Christ

Germany

Yu Jo Chua

Australia

Seng-Kee Chuah

Taiwan

Chen Shuan Chung

Taiwan

Michele Cicala

Italy

Rachele Ciccocioppo

Italy

Sandra Ciesek

Germany

Marilisa Citton

Italy

Mauro Cives

Italy

Marguerite Clyne

Ireland

Irene Coati

Italy

Mehmet Coban

Turkey

Gareth Corbett

UK 
Francesca Cordero

Italy

Maura Corsetti

Belgium

Chris Corton

USA

Luís Costa Matos

Portugal

Melinda Coughlan

Australia

Thorsten Cramer

Germany

Cesare Cremon

Italy

Javier Crespo

Spain

Tim Cross

UK

Alessandro Cucchetti

Italy

Adrian Cummins

Australia

Rosario Cuomo

Italy

Douglas Curran-Everett

USA

Srinivasan Dasarathy

USA

Ilva Daugule

Latvia

Eric Davies

USA

Andrew Day

Australia

Paola De Angelis

Italy

Nicola De Bortoli

Italy

Roberto De Franchis

Italy

Richarda De Voer

Netherlands

Evelien Dekker

Netherlands
Jean Charles Delchier

France

Christine Delporte

Belgium

Renumathy Dhanasekaran

USA

Puneet Dhar

India

John Dillon

UK

Phil Dinning

Australia

Enric Domingo

UK

Quanjiang Dong

China

Gabriella D'Orazi

Italy

Richard Douard

France

Jacqueline Doyle

UK

Christos Dr Zouboulis

Germany

Ignat Drozdov

UK

Hanna Dückers-Horst

Germany

Philippe Ducrotte

France

Pradeep Dudeja

USA

Dan Dumitrascu

Romania

Cuong Duong

Australia

Lynn Dustin

UK

Adam Dziki

Poland

Sanna Edelman

Finland

Piotr Eder

Poland
Robert Eferl

Austria

Laurent Ehrlich

USA

Nikos Eleftheriadis

Greece

Abdul Elkadri

Canada

Pierre Ellul

Malta

Magdy El-Salhy

Norway

Christoph Elsing

Germany

Cathy Eng

USA

Arzu Ensari

Turkey

Meira Epplein

USA

Askin Erdogan

Turkey

Elsa Fabbretti

Slovenia

Jan Fallingborg

Denmark

Klaudia Farkas

Hungary

Per G Farup

Norway

Peter Ferenci

Austria

Conrado M Fernandez Rodriguez Spain

Jose Fernandez-Cebrian

Spain

Christian Fingas

Germany

Gionata Fiorino

Italy

Gabor Firneisz

Hungary

Wolfgang Fischer

Germany 
P. Marco Fisichella

USA

Reidar Fossmark

Norway

Giampiero Francica

Italy

Martin Freeman

USA

Michael Freemark

USA

Shunjun Fu

China

Ho Fuang

Malaysia

Shiki Fujino

Japan

David Fuks

France

Mathurin Fumery

France

Akihiro Funakoshi

Japan

Erwin Gäbele

Germany

Gary Galante

Canada

Giovanni Galati

Italy

Francesca Galeazzi

Italy

Enrico Galmozzi

Italy

Shivanand Gamanagatti

India

Rita Garcia Martinez

Spain

Pramod Garg

India

Manuela Gariboldi

Italy

Zhongming Ge

USA

Krisztina Gecse

Hungary
Fernand-Pierre Gendron

Canada

Uday Ghoshal

India

Cinzia Giacometti

Italy

Catia Giovannini

Italy

Robert Gish

USA

Shannon Glaser

USA

Weihua Gong

China

Elisabetta Goni

Italy

Jeroen Goos

Netherlands

Melita Gordon

UK

Ramon Gorter

Netherlands

Gunnar Gothberg

Sweden

Peter Green

USA

Jennifer Griffin

Canada

Shannon Griffin

USA

Mehmet Haberal

Turkey

Luciana Haddad

Brazil

Chad Hall

USA

Barry Hall

Ireland

Heather Hampel

USA

Yuyan Han

USA

Kenichi Harada

Japan
Elaine Harkness

UK

Muhammad Hasan

USA

Cesare Hassan

Italy

Benjamin Heidrich

Germany

Fernando Herbella

Brazil

Tiberiu Hershcovici

USA

Toshifumi Hibi

Japan

Hatanaka Hisashi

Japan

Joerg Hoffmann

Germany

Christoph Höner Zu Siederdissen

Germany

Ping-Hsin Hsieh

Taiwan

Jan-Sing Hsieh

Taiwan

Chien-Ning Hsu

Taiwan

Pin-I Hsu

Taiwan

Qin Huang

USA

Patrick Hughes

Australia

Yee Tak Hui

Hong Kong

Hung-Hsu Hung

Taiwan

Richard Hunt

Canada

Christine M. Hunt

USA

Musharraf Husain

India

Jong Jin Hyun

Korea, South 
Marietta Iacucci

Canada

Gianluca Ianiro

Italy

Sergio Iannazzo

Italy

Gasim Ibrahim

Saudi Arabia

Tsukasa Ikeura

Japan

Jun Imamura

Japan

Akira Imoto

Japan

Patrick Ingiliz

Germany

Sergejs Isajevs

Latvia

Claudio Isella

Italy

Genichiro Ishii

Japan

Jeremie Jacques

France

Khaled Jadallah

Jordan

Jana Jandova

USA

Julien Jarry

France

Irma Järvelä

Finland

Peter Jepsen

Denmark

Ran Jin

USA

Tatehiro Kagawa

Japan

Rakesh Kalapala

India

Frank Kallenberg

Netherlands

Julia Kälsch

Germany
Nobuhiko Kamada

USA

Tatsuo Kanda

Japan

Arne Kandulski

Germany

Kenitiro Kaneko

Japan

Eun-Suk Kang

Korea, South

Wei-Yu Kao

Taiwan

Konstantinos Karmiris

Greece

David Katzka

USA

Yasunari Kawabata

Japan

Robin Kelley

USA

Paul Kelly

UK

Kawada Kenro

Japan

Arif Ali Khan

India

Alexander Khoruts

USA

Jan Kielstein

Germany

Kyoung Kim

Korea, South

Ingorn Kimkong

Thailand

Jens Kittner

Germany

Anne Knudsen

Denmark

Masaaki Kobayashi

Japan

S Komatsu

Japan

Tine Kopp

Denmark
Uri Kopylov

Canada

Jay Kostman

USA

Anastasios Koulaouzidis

UK

Aleksander Krag

Denmark

Rajesh Krishnamoorthi

USA

Suneeta Krishnareddy

USA

Chikara Kunisaki

Japan

Chao-Hung Kuo

Taiwan

Juozas Kupcinskas

Lithuania

Vincenzo La Mura

Italy

Stephen Lagana

USA

Terry Lairmore

USA

Yan Lam

Australia

Laura Lamberti

USA

Dora Lam-Himlin

USA

Frank Lammert

Germany

Linda Law

USA

Cedric Le May

France

Hye-Seung Lee

USA

Tsung-Chun Lee

Taiwan

Jae-Hoon Lee

Korea, South

Teng-Yu Lee

Taiwan 
Philippe Lehours

France

Maureen Leonard

USA

Maria Liz Leoz

Spain

Cosmas Rinaldi Lesmana

Indonesia

Cynthia Levy

USA

David Lewin

USA

Stephen Lewis

UK

James Lewis

USA

Yue Li

China

Chris Liacouras

USA

Wei-Chih Liao

Taiwan

Lev Lichtenstein

Israel

Paolo Limongelli

Italy

Lian-Jie Lin

China

Alexander Link

Germany

Jyh-Ming Liou

Taiwan

Tao Liu

Australia

Huwei Liu

China

Gin-Ho Lo

Taiwan

Wai-Kit Lo

USA

Josefa Leon Lopez

Spain

Vicente Lorenzo-Zúñiga García Spain
Albert Lowenfels

USA

Ligong Lu

China

Yongke Lu

USA

Claudio Luchini

Italy

Jonas Ludvigsson

Sweden

Rashid Lui

Hong Kong

Jiing-Chyuan Luo

Taiwan

Stefan Lüth

Germany

Francesco Luzza

Italy

Benjamin Maasoumy

Germany

Laurence Macia

Australia

Giovanni Maconi

Italy

Kaushal Madan

India

Varocha Mahachai

Thailand

Avik Majumdar

UK

Govind Makharia

India

Richard Makins

UK

Mariano Malaguarnera Italy

Shahid Malik

USA

Rosalie Mallant-Hent

Netherlands

Giuseppe Malleo

Italy

Andrew Mallett

Australia
Vivek Mangla

India

Murugu Manickam

USA

Hendrik Manner

Germany

Giovanni Marchegiani

Italy

Giulio Marchesini

Italy

Adil Mardinoglu

Sweden

Alberto Mariani

Italy

Monica Mars

Netherlands

Irene Martinucci

Italy

Riruke Maruyama

Japan

Hitoshi Maruyama

Japan

Marco Marzioni

Italy

Daiuske Masuda

Japan

Satohiro Masuda

Japan

Kei Matsueda

Japan

Dimitrios Mavridis

Greece

Jens Mayer

Germany

Steven Mcelroy

USA

Thomas Mcgarrity

USA

Cristina Meazza

Italy

Makoto Meguro

Japan

Arianeb Mehrabi

Germany 
Fanyin Meng

USA

Shahin Merat

Iran

Ahmed Messallam

USA

Evangelos Messaris

USA

James Mezhir

USA

Pal Miheller

Hungary

Antonina Mikocka-Walus

Australia

Danilo Miskovic

UK

Benjamin Misselwitz

Switzerland

Tatsuya Miyazaki

Japan

Meir Mizrahi

Israel

Mansour Mohamadzadeh

USA

Paola Molteni

Italy

Giuseppe Montalto

Italy

Senador Moran Sanchez

Spain

F Motoi

Japan

Stefan Mueller-Lissner

Germany

Chris Mulder

Netherlands

Mohamed Mutalib

UK

Alessio Naccarati

Italy

Sellama Nadifi

Morocco

Kenji Nagahama

Japan
Hayato Nakagawa

Japan

Atsushi Nakajima

Japan

Masanao Nakamura

Japan

Jun Nakamura

Japan

Cindy Neuzillet

France

Lorenzo Nicolè

Italy

Abdelrahman Nimeri

United Arab Emirates

Madunil Niriella

Sri Lanka

Alireza Norouzi

Iran, Islamic Republic Of

Thiago Nunes

Brazil

Ichiro Oda

Japan

Takeshi Ogura

Japan

Yuki Ohya

USA

Shiro Oka

Japan

Hussein Okasha

Egypt

Kazuichi Okazaki

Japan

Monica Oleastro

Portugal

Nicholas Onaca

USA

David Ong

Singapore

Shoko Ono

Japan

Sofia Ornelas-Arroyo

Mexico

Benjamin Otto

Germany
Richard Owen

UK

Gokben Ozbey

Turkey

Fabio Pace

Italy

Amanda Page

USA

Sujoy Pal

India

Shanthi Palaniappan

Malaysia

Senthilnathan Palanisamy

India

Giovanni Pallabazzer

Italy

Vincenzo Palmieri

USA

Gopanandan Parthasarathy

USA

Robert Partyka

Poland

Eleonora Patsenker

Switzerland

Natalia Pedersen

Denmark

Gianmaria Pennelli

Italy

Luit Penninga

Denmark

Sharon Perrella

Australia

Raffaele Pezzilli

Italy

Phoebe Phillips

Australia

Guillaume Piessen

France

Alejandro Piscoya

Peru

Joseph Pisegna

USA

Salvador Pita-Fernández

Spain 
Michael Pitiakoudis

Greece

Marco Pizzi

Italy

Lorenzo Polimeno

Italy

Kevin Pollock

UK

Hans-Christian Pommergaard

Denmark

Alina Popp

Finland

Dario Porett

Italy

Hasmukh Prajapati

USA

Jan Preiss

Germany

Timothy Price

Australia

Jesus Prieto

Spain

Chethan Puttarajappa

Panama

M. Masudur Rahman

Bangladesh

H Ramesh

India

Jens Rasenack

Germany

Norman Ratcliffe

UK

Matteo Ravaioli

Italy

Ratha Ray

USA

Pradeep Rebala

India

Helen Reeves

UK

Norelle Reilly

USA

Helen Remotti

USA
Johannes Rey

Germany

Silvia Ribback

Germany

Claudio Ricci

Italy

Thomas Ritz

Germany

Mar Riveiro-Barciela

Spain

Juan Carlos Roa

Chile

Ian Roberts-Thomson

Australia

Xavier Roblin

France

Michael Roerecke

Canada

Yishai Ron

Israel

Emanuele Rondonotti Italy

Jonas Rosendahl

Germany

Christophe Rosty

Australia

Manolis Roulis

USA

Andrew Rowland

Australia

Enrica Rumiato

Italy

Salvatore Russo

Italy

Akiko Saka

Japan

Efstratios Saliakellis

Greece

Amanda Salis

Australia

Sundeep Saluja

India

Madhusudhan Sanaka

USA
Olof Sandström

Sweden

Angelo Sangiovanni

Italy

Roberto Santambrogio

Italy

Deborah Saraggi

Italy

Samantha Sarcognato Italy

Giovanni Sarnelli

Italy

Stefan Sauerland

Germany

Jeremy Schaefer

USA

David Schaeffer

Canada

Christopher Schalchta

Canada

Joern M. Schattenberg

Germany

Annalisa Schiepatti

Italy

Robert Schierwagen

Germany

Margaret Schneider

Canada

Jonathan Schwartz

USA

Andrada Seicean

Romania

Neil Shah

UK

Muhammed Sherid

USA

Oren Shibolet

Israel

Yasuhiro Shirakawa

Japan

Elham Shirvani

Germany

Ivy Shiue

UK 
Shailesh Shrikhande

India

Sadiq Sikora

India

Siddharth Singh

USA

Vikesh Singh

USA

Harkirat Singh

USA

Manish Singla

USA

Marianne Sinn

Germany

Venkatramani Sitaram

India

Mamata Sivagnanam

USA

Ali Kemal Sivrioglu

Turkey

Antonio Solins

Italy

Amornyotin Somchai

Thailand

Ming Song

USA

Bridget Southwell

Australia

Margarida Souto-Carneiro

Portugal

Ioan Sporea

Romania

Ankur Srivastava

UK

Amitabh Srivastava

USA

Peter Stanich

USA

Horia Stefanescu

Romania

Costin Teodor Streba

Romania

Venkat Subramanian

UK
Mitsushige Sugimoto

Japan

Xueying Sun

New Zealand

Kai-Feng Sung

Taiwan

Takuya Suzuki

Japan

Ingvar Syk

Sweden

Wing-Kin Syn

UK

Shun Fung Sze

Hong Kong

Sheryl Szeinbach

USA

Zoltan Szepes

Hungary

James Tabibian

USA

Frank Tacke

Germany

Wei-Chen Tai

Taiwan

Yoshitsugu Tajima

Japan

Masanobu Takahashi

Japan

Shao-Tao Tang

China

Elliot Tapper

USA

Giovanni Tarantino

Italy

Alex Tarr

UK

Daynijela Tasic

Serbia

Julia Tchou

USA

Sooraj Tejaswi

USA

Koji Teramoto

Japan
Traci Testerman

USA

Zhen Tian

USA

Hans Tillmann

USA

Kazutomo Togashi

Japan

Michele Tonerini

Italy

Francesco Tovoli

Italy

Caroline Trang

France

Jonel Trebicka

Germany

Guglielmo Trovato

Italy

Konstantinos Tsimogiannis

Greece

Yasuhiro Tsuda

Japan

Yishito Tsushima

Japan

Pamela Tuma

USA

Nesrin Ugras

Turkey

Holm Uhlig

UK

Shahid Ullah

Australia

Bella Ungar

Israel

Dino Vaira

Italy

Barbara Valdivia-Correa

Mexico

Leo Van Rossum

Netherlands

Niels Vande Casteele

USA Minor Outlying Islands

Sina Vatandoust

Australia 
Nereo Vettoretto

Italy

Alessandro Vitale

Italy

Jiannis Vlachogiannakos

Greece

Jasper Vleugels

Netherlands

Pavel Vodicka

Czech Republic

Thomas Von Hahn

Germany

Ulrike Von-Arnim

Germany

Mihael Vucur

Germany

Veronika Vymetalkova

Czech Republic

Timothy Wade

USA

Mihir Wagh

USA

Homan Wai

USA

Maximilian Waldner

Germany

Liangjing Wang

China

Fusheng Wang

China

David Wang

USA

Chih-Yuan Wang

Taiwan

Jing-Houng Wang

Taiwan
David G Watt

UK

Malte Wehmeyer

Germany

Shu-Chen Wei

Taiwan

Martin Weltman

Australia

Thomas Wex

Germany

J. Patrick Whelan

USA

Edyta Wieczorek

Poland

Kathryn Williams

Australia

Christopher Williams

USA

Caroline Wilson

UK

Andrzej Wincewicz

Poland

Carl Wingren

Sweden

Jean Winter

USA

Piotr Witkowski

USA

Robert Wong

USA

Daniel Worthley

Australia

Dan Worthley

Australia

Alexander Wree

USA
Keng-Liang Wu

Taiwan

Yinglian Xiao

China

Javed Yakoob

Pakistan

Shih-Cheng Yang

Taiwan

Pw Yeh

Taiwan

Chih-Hsun Yi

Taiwan

Takehiko Yokobori

Japan

Xin Yongning

China

Ying Yuan

China

Arseniy Yuzhalin

UK

Shira Zelber-Sagi

Israel

Patrizia Zentilin

Italy

Lin Zhang

China

Qiang Zhang

China

Jingmin Zhao

China

Shusen Zheng

China

Lixin Zhu

China 